\title{
UNILATERAL OEDEMA OF THE LOWER LIMB CAUSED BY AN OSTEOCHONDROMA
}

\author{
M. G. MATTHEWS
}

Case 1. A 37-year-old housewife noticed gradual swelling of her left leg over a period of approximately one year. She had no other symptoms. At the age of 24 she had undergone subtotal thyroidectomy for toxic goitre, and was taking replacement thyroxine. She was also taking propranolol for mild hypertension. She appeared fit and clinically euthyroid.

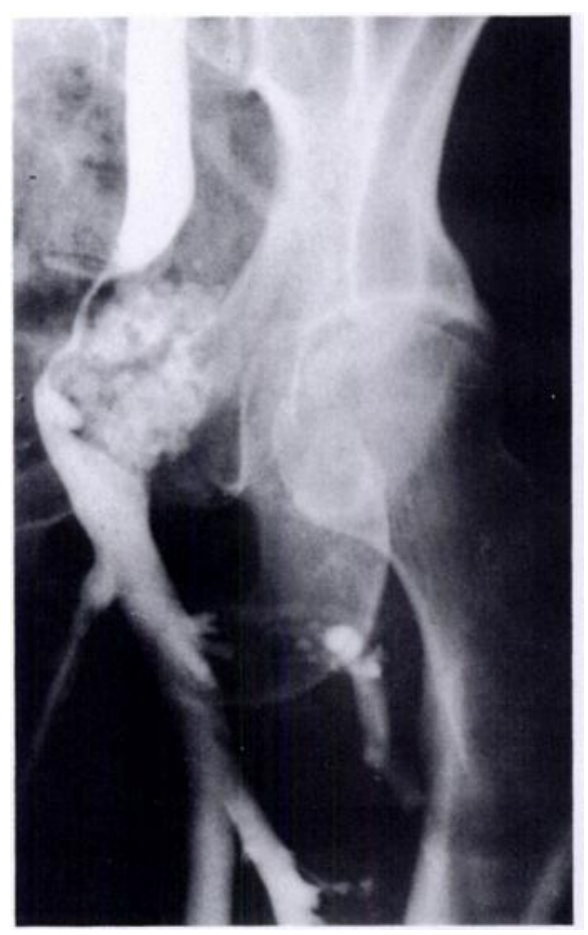

Fig. 1

There was uniform swelling of the left lower limb from the toes to the inguinal region. The girth of the left calf was $2 \mathrm{~cm}$ greater than that of the right. Dilated venules and arbourising telangiectasia were noted over the front of the leg. A localised swelling, firm and immobile, was discovered in the left inguinal region; the skin appeared normal over this swelling and was not tethered. Plain radiographs showed a calcified lesion

M. G. Matthews, FRCS, Orthopaedic Registrar Newham General Hospital, Glen Road, London E13 8SL, England. (C) 1987 British Editorial Society of Bone and Joint Surgery $0301-620 X / 87 / 2 \mathrm{R} 18 \$ 2.00$ related to the left superior pubic ramus, and venography demonstrated compression of the left iliac vein by the lesion (Fig. 1).

At operation, biopsy and frozen section were performed, followed by complete excision of the tumour. Histologically it proved to be a benign osteochondroma. The patient made an uncomplicated recovery, and after

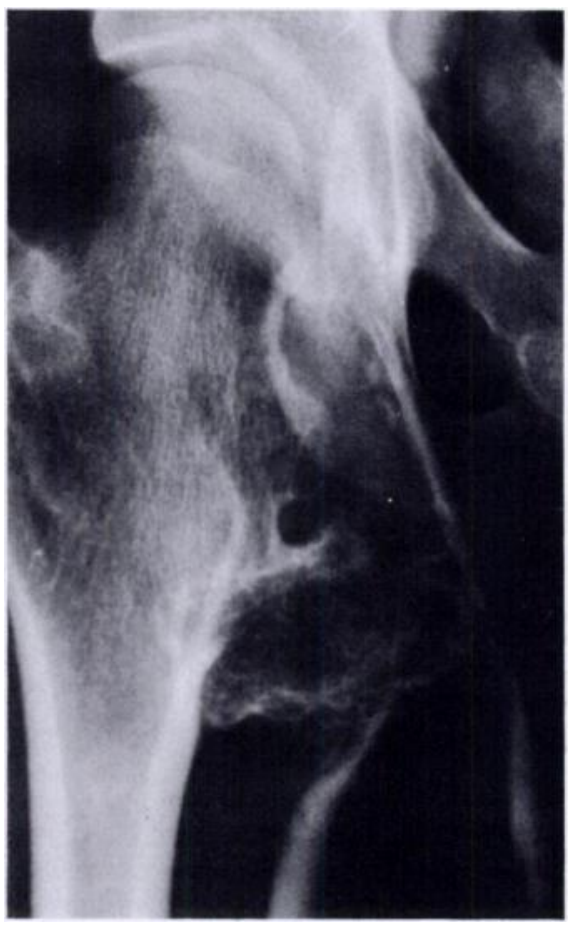

Fig. 2

one month the left leg was equal in girth to the right. Four years later, clinical examination and radiography showed no evidence of recurrence.

Case 2. A 15-year-old schoolboy gave a history of swelling of the right leg since childhood. He had also noticed a painless lump developing in the right groin over the past two years. There was a known family history of diaphyseal aclasis. The whole of the right lower limb was oedematous. At mid-thigh the girth was $6 \mathrm{~cm}$ greater than the left, and at the calf it was $3 \mathrm{~cm}$ greater. A bony hard lump was palpable in the right femoral triangle. The pulsating femoral artery could be felt crossing superficially over it. Plain radiographs showed a 
large exostosis arising from the right lesser trochanter. Venography showed extrinsic compression of the femoral vein distal to the saphenofemoral junction (Fig. 2).

Exploration was performed via an anterior approach. The femoral vessels and nerve were dissected off the dome of the tumour. The sartorius muscle was divided to provide adequate exposure. The tumour was excised after transecting its neck with an osteotome. Histology confirmed a benign osteochondroma with no sarcomatous change. After operation, the leg slowly reduced in girth and at nine months the thighs were equal, though the right calf remained $2 \mathrm{~cm}$ greater than the left. At 18 months the legs were symmetrical.

Discussion. Acquired unilateral oedema is commonly due to thrombophlebitis or deep venous thrombosis. When oedema of any fluid overload state is just beginning, it is not infrequently unilateral. More rarely, oedema may result from obstruction of veins or lymphatics by malignant neoplasms. Prostatic carcinoma predominates in men, and lymphoma in women.

Unilateral oedema of the lower limb caused by an osteochondroma does not seem to have been reported previously. In each of the two cases described excision of the tumour resulted in complete resolution of the oedema, tending to confirm that the oedema has been caused by extrinsic compression of the deep veins by this benign tumour. It would appear that we can expect surgery to achieve a permanent cure in such patients.

I am grateful to Mr E. M. Downes, Mr M. C. Mason, and Mr H. S. Winsey for permitting me to report on their patients who were treated at Singleton and Morriston Hospitals, Swansea, West Glamorgan. Dr $\mathrm{N}$. Powell and Dr M. White performed radiographic studies and Dr O. G. Williams carried out histological investigation.

\title{
LONGITUDINAL RUPTURE OF THE PERONEUS BREVIS TENDON
}

\author{
EILIF LARSEN
}

Two cases of longitudinal rupture of the peroneus longus tendon are reported.

Case 1. A 31-year-old footballer had severely sprained his right ankle some 10 years earlier. He had been treated in plaster for four weeks but, because of pain and instability, had discontinued all sports activities. Radiographically the right ankle showed a talar tilt of $6.5^{\circ}$ and an anterior rotational instability of $2.5 \mathrm{~mm}$, compared to the left with $3.0^{\circ}$ and $0.5 \mathrm{~mm}$, respectively.

At operation the anterior talofibular ligament was fibrotic and elongated whereas the calcaneofibular and posterior talofibular ligaments were normal. The peroneal tendon sheaths looked oedematous. The peroneus

E. Larsen, MD, Orthopaedic Department

Gentofte Hospital, University of Copenhagen, Niels Andersensvej 65, DK-2900 Hellerup, Denmark.

Correspondence to Dr E. Larsen, Lyngviguej 14, DK-2720 Vanløose, Denmark.

(C) 1987 British Editorial Society of Bone and Joint Surgery $0301-620 \mathrm{X} / 87 / 2 \mathrm{R} 11 \$ 2.00$ brevis tendon had been ruptured longitudinally over a length of $5 \mathrm{~cm}$ just behind the lateral malleolus and below the peroneal retinaculum. The tendon was somewhat thickened but not necrotic. It was divided further proximally and its distal anterior half was used to perform a modified Evans procedure, which stabilised the ankle joint laterally. A below-knee plaster was worn for six weeks. At follow-up 23 months later the patient had returned to football, his only complaint being a feeling of tiredness in the lateral crural compartment after prolonged activity.

Case 2. A 43-year-old male tennis player complained that for over a year, since an ankle sprain, he had had pain, swelling and ankle instability when playing and running on uneven ground. Several years earlier he had sustained a number of severe ankle sprains. He had discontinued all sport. Clinically, there was swelling below and behind the lateral malleolus, with local tenderness and pain made worse by dorsiflexion. Stress radiographs showed no instability. 